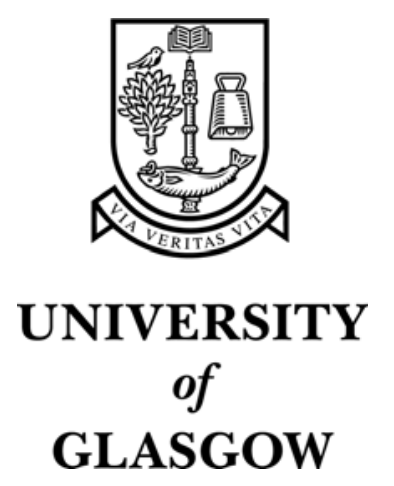

Paul, M.C. and Rees, D.A.S. and Wilson, M. (2005) Influence of higher order effects on the linear wave instability of vertical free convective boundary layer flow. International Journal of Heat and Mass Transfer 48(3-4):pp. 809-817.

http://eprints.gla.ac.uk/3329/ 


\title{
THE INFLUENCE OF HIGHER ORDER EFFECTS ON THE LINEAR INSTABILITY OF VERTICAL FREE CONVECTIVE BOUNDARY LAYER FLOW
}

\author{
Manosh C. Paul ${ }^{\dagger}$, D. Andrew S. Rees*, Michael Wilson \\ Department of Mechanical Engineering, University of Bath, \\ Claverton Down, Bath, BA2 7AY, UK
}

\begin{abstract}
We examine the stability of free convective boundary layer flow over a vertical heated flat plate with respect to two-dimensional wave disturbances. In particular we determine the effect of the overall external geometry on the stability criterion. The fluid domain is taken to be bounded by two semi-infinite flat plates forming a wedge of angle $\alpha$. The vertical plate is held at a uniform hot temperature while the other is either insulated or is held at the ambient temperature of the fluid. The basic flow used in the analysis is a two-term boundary-layer approximation using the method of matched asymptotic expansions. A modified version of the Keller-box method is used to solve the linearised wave-disturbance equations numerically. The neutral curves have been delineated for different values of wedge angle, $\alpha$, where the working fluids are water and air. We find that the critical distance from the leading edge beyond which disturbances grow is strongly dependent on $\alpha$, and this suggests that the external geometry of the fluid domain exerts a considerable influence on stability criteria.
\end{abstract}

Keywords: Free convection; Boundary layer; Wave instability; Higher order effects

\section{Introduction.}

There is a very large literature associated with the analysis of thermal boundary layer instabilities. A comprehensive review of the wider topic of thermal boundary layer flows was reviewed by Gebhart [1]. For the vertical free convection boundary layer the basic flow arises from the presence of buoyancy forces, but the primary instability is well-known to be hydrodynamic in origin. Thus this boundary layer shares some stability characteristics with the Blasius boundary layer and is subject to a primary mode of instability in the form of two-dimensional waves travelling in the streamwise direction (see Nachtsheim [2], Heiber \& Gebhart [3], Haaland \& Sparrow [4], Godaux \& Gebhart [5] and Szewczyk [6]). The experimental work undertaken by Szewczyk [6] also demonstates that there is

$\dagger$ Current address: Department of Mechanical Engineering, University of Glasgow, Glasgow G12 8QQ, Scotland

* Corresponding author. Email: D.A.S.Rees@bath.ac.uk 
a three-dimensional secondary instability mechanism which occurs further downstream; to date there are no theoretical studies of this particular aspect.

Most of the earlier theoretical studies of the linear stability characteristics of boundary layer flows use the parallel flow approximation. In these analyses the basic flow is assumed either to be parallel, or, more generally, sufficiently slowly varying in the streamwise direction that such variations may be ignored. However, Haaland and Sparrow [4,7] allowed for the nonparallelism which is inherent in free convective flows by incorporating $x$-dependent terms in their disturbance equations, which are the equivalent to the OrrSomerfeld equations. In [4], they conclude that such nonparallel effects serve to increase the neutral distance relative to that obtained by Nachtsheim [2] who used parallel flow theory.

All of the above authors used the leading order boundary layer flow as the basic flow whose stability was considered. Strictly speaking, the leading order boundary layer flow forms part of an asymptotic theory which is valid in the asymptotic limit of large distances from the leading edge. However, it remains fairly accurate at moderate distances from the leading edge. In the present paper we use a more accurate representation of the flow in the boundary layer by determining the influence on the boundary layer of the flow which is induced in the region external to the boundary layer. The analysis of the interaction of the boundary layer flow and the external region can only be made by invoking the boundary layer approximation and subsequent application of the method of matched asymptotic expansions. Again this means that the distance from the leading edge $(x)$ should be asymptotically large, but the result of the ensuing stability analysis yields a finite value of $x$. Thus corrections to the leading boundary layer flow are not necessarily vanishingly small when using higher order boundary layer theory to describe the basic flow at the marginally stable value of $x$.

Such a technique has been used recently by Storesletten and Rees [8] who considered the the onset of instabilities in the form of streamwise vortices which occur in the thermal boundary layer induced by a heated inclined surface embedded in a saturated porous medium. Those authors discovered that stability criteria are very highly dependent on the flow field which is external to the boundary layer.

In the present paper we extend the technique of [4] by applying the method of analysis of Storesletten and Rees [8] to the problem of the wave instability of thermal boundary layer flow of a clear fluid from a vertical surface. As in [8] we assume that the fluid is bounded by two semi-infinite flat plates. One of these is the vertical heated surface while the other is either insulated or at the ambient temperature of the medium. The surfaces form a wedge of angle $\alpha$, and the basic flow is obtained using the method of matched asymptotic expansions. Although the leading order boundary flow is independent of $\alpha$, further terms are functions of $\alpha$. The full disturbance equations are reduced to ordinary 


\section{Nomenclature}

$\begin{array}{ll}A_{0} & \text { constant } \\ d & \text { natural lengthscale } \\ F, G, H & \text { disturbances } \\ F_{0}, H_{0} & \text { leading order boundary layer solutions } \\ F_{1}, H_{1} & \text { first order boundary layer solutions } \\ g & \text { gravitational acceleration } \\ k & \text { wavenumber } \\ P & \text { dynamic pressure } \\ P r & \text { Prandtl number } \\ r & \text { nondimensional radial distance from origin } \\ \bar{r} & \text { radial distance from origin } \\ t & \text { nondimensional time } \\ \bar{t} & \text { time } \\ T & \text { temperature of the fluid } \\ u, v & \text { nondimensional fluid velocities in the } x, y \text { directions } \\ \bar{u}, \bar{v} & \text { fluid velocities in the } \bar{x}, \bar{y} \text { directions } \\ U & \text { natural velocity scale } \\ x, y & \text { nondimensional Cartesian coordinates } \\ \bar{x}, \bar{y} & \text { Cartesian coordinates }\end{array}$

Greek symbols

$\begin{array}{ll}\alpha & \text { wedge angle } \\ \beta & \text { coefficient of thermal expansion } \\ \gamma & \text { scaled wedge angle } \\ \Delta T & \text { temperature scale } \\ \epsilon & \text { small value } \\ \eta & \text { similarity variable } \\ \theta & \text { scaled temperature } \\ \kappa & \text { thermal diffusivity } \\ \lambda & \text { complex exponential growth rate } \\ \nu & \text { fluid viscosity } \\ \rho & \text { density of fluid } \\ \phi & \text { angular coordinate } \\ \psi & \text { streamfunction } \\ \Psi & \text { streamfunction in outer region } \\ \omega & \text { vorticity }\end{array}$

Superscripts and subscripts

$\begin{array}{ll}B & \text { basic flow } \\ c & \text { critical } \\ D & \text { disturbance } \\ I & \text { imaginary part } \\ n & \text { normal derivative } \\ R & \text { real part } \\ w & \text { wall or heated surface } \\ \infty & \text { ambient conditions } \\ 1 & \text { derivatives with respect to } \eta\end{array}$


differential form in the usual way and are solved numerically to determine how the shape of the overall fluid domain affects the stability criterion.

\section{Governing equations.}

We consider the two-dimensional free convective boundary layer flow from a vertical semi-infinite heated plate as shown in Fig. 1. Apart from the fluid Prandtl number, the only other parameter is the wedge angle, $\alpha$. A Cartesian frame of reference is chosen, where the $x$-axis is aligned vertically with the heated surface and the $y$-axis is perpendicular to this.

The system of equations which describe the free convection from a vertical plate is taken to be the Navier-Stokes and energy equations. For unsteady two dimensional flow subject to the Boussinesq approximation this system is written in the dimensional form

$$
\begin{gathered}
\bar{u}_{\bar{x}}+\bar{v}_{\bar{y}}=0 \\
\bar{u}_{\bar{t}}+\bar{u} \bar{u}_{\bar{x}}+\bar{v} \bar{u}_{\bar{y}}=-\frac{1}{\rho} P_{\bar{x}}+\nu\left(\bar{u}_{\bar{x} x}+\bar{u}_{\bar{y} y}\right)+g \beta\left(T-T_{\infty}\right), \\
\bar{v}_{\bar{t}}+\bar{u}_{\bar{v}_{\bar{x}}}+\bar{v} \bar{v}_{\bar{y}}=-\frac{1}{\rho} P_{\bar{y}}+\nu\left(\bar{v}_{\bar{x} x}+\bar{v}_{\bar{y} y}\right), \\
T_{\bar{t}}+\bar{u} T_{\bar{x}}+\bar{v} T_{\bar{y}}=\kappa\left(T_{\bar{x} x}+T_{\bar{y} y}\right) .
\end{gathered}
$$

Here $\bar{u}$ and $\bar{v}$ are the velocity components in the $\bar{x}$ and $\bar{y}$-directions, respectively, $\bar{t}$ is time, $P$ is the dynamic pressure, $T$ is the temperature, $g$ is the gravitational acceleration in the negative $\bar{x}$-direction, $\rho$ is the density of the fluid, $\nu$ is the kinematic viscosity, $\kappa$ is the diffusivity and $\beta$ is the coefficient of thermal expansion.

The boundary conditions for Eqs. (1)-(4) for the case of an isothermal plate are:

$$
\begin{gathered}
\bar{u}=\bar{v}=0, \quad T=T_{w} \quad \text { at } \quad \bar{y}=0, \quad \bar{x} \geq 0 \\
\bar{u}=\bar{v}=0, \quad \text { and either } \quad T_{n}=0 \text { or } T=T_{\infty} \text { on the second surface, }
\end{gathered}
$$

and

$$
T \rightarrow T_{\infty} \quad \text { as } \quad \bar{r}=\left(\bar{x}^{2}+\bar{y}^{2}\right)^{1 / 2} \rightarrow \infty,
$$

where $T_{w}$ is the temperature of the plate and $T_{\infty}$ is the ambient temperature of the fluid with $T_{w}>T_{\infty}$, and $n$ as a subscript denotes the normal derivative. Eqs. (1)-(7) may be nondimensionalized using the following transformations:

$$
(\bar{x}, \bar{y})=d(x, y), \quad \bar{t}=(d / U) t, \quad(\bar{u}, \bar{v})=U(u, v) \quad T-T_{\infty}=\left(T_{w}-T_{\infty}\right) \theta,
$$

where the natural length and velocity scales, $d$ and $U$, are given by,

$$
d=\left(\frac{\nu^{2}}{g \beta \Delta T}\right)^{1 / 3}, \quad U=(g \beta \nu \Delta T)^{1 / 3} .
$$


A further simplication is afforded by the introduction of a streamfunction, $\psi$, according to $u=\psi_{y}$ and $v=-\psi_{x}$. Eqs. (1)-(4) now become

$$
\begin{gathered}
\nabla^{4} \psi=\nabla^{2} \psi_{t}+\psi_{y} \nabla^{2} \psi_{x}-\psi_{x} \nabla^{2} \psi_{y}-\theta_{y}, \\
\frac{1}{\operatorname{Pr}} \nabla^{2} \theta=\theta_{t}+\psi_{y} \theta_{x}-\psi_{x} \theta_{y},
\end{gathered}
$$

where $\operatorname{Pr}=\nu / \kappa$ is the Prandtl number. The corresponding boundary conditions may be written as follows

$$
\begin{gathered}
\psi_{y}=\psi=0, \quad \theta=1 \quad \text { at } \quad y=0, \quad x \geq 0 \quad[\text { i.e. } \phi=0] \\
\psi=\psi_{n}=0, \quad \text { and either } \quad \theta_{n}=0 \quad \text { or } \quad \theta=0 \quad \text { on } \phi=\alpha,
\end{gathered}
$$

and

$$
\theta \rightarrow 0 \quad \text { as } \quad r=\left(x^{2}+y^{2}\right)^{1 / 2} \rightarrow \infty .
$$

\section{Basic flow analysis.}

In this section we determine the two-term solution of the undisturbed basic boundary layer flow using the method of matched asymptotic expansions. The basic flow, which we denote by the subscript $B$, is steady and two-dimensional and it satisfies the equations

$$
\begin{gathered}
\nabla^{2} \psi_{B}=\omega_{B} \\
\nabla^{2} \omega_{B}=\frac{\partial \psi_{B}}{\partial y} \frac{\partial \omega_{B}}{\partial x}-\frac{\partial \psi_{B}}{\partial x} \frac{\partial \omega_{B}}{\partial y}-\frac{\partial \theta_{B}}{\partial y} \\
\frac{1}{P r} \nabla^{2} \theta_{B}=\frac{\partial \psi_{B}}{\partial y} \frac{\partial \theta_{B}}{\partial x}-\frac{\partial \psi_{B}}{\partial x} \frac{\partial \theta_{B}}{\partial y}
\end{gathered}
$$

where $\psi_{B}$ is the basic flow streamfunction and $\omega_{B}$ denotes the basic vorticity, as defined by (15). Eqs. (15)-(17) are to be solved subject to the boundary layer approximation. Therefore we introduce the following expansions for the streamfunction and temperature in the boundary layer region,

$$
\begin{gathered}
\psi_{B}=x^{3 / 4} F_{0}(\eta)+F_{1}(\eta)+\cdots \\
\theta_{B}=H_{0}(\eta)+x^{-3 / 4} H_{1}(\eta)+\cdots
\end{gathered}
$$

where the similarity variable $\eta$ is given by

$$
\eta=y / x^{1 / 4},
$$

and we apply the boundary layer approximation wherein we assume that $x \gg y$. The equations for the zeroth order terms, $F_{0}$ and $H_{0}$, correspond to the classical problem:

$$
F_{0}^{\prime \prime \prime}+\frac{3}{4} F_{0} F_{0}^{\prime \prime}-\frac{1}{2} F_{0}^{\prime} F_{0}^{\prime}+H_{0}=0,
$$




$$
H_{0}^{\prime \prime}+\frac{3}{4} \operatorname{Pr} F_{0} H_{0}^{\prime}=0,
$$

subject to

$$
F_{0}(0)=F_{0}^{\prime}(0)=0, H_{0}(0)=1, \quad F_{0}^{\prime}(\infty)=H_{0}(\infty)=0 .
$$

Given that solutions to Eq. (22) decay exponentially to zero, the region outside the boundary layer is isothermal and we may ignore the temperature field in this potential flow region. If we denote by $\Psi$ the outer-region streamfunction, then we may expand it according to

$$
\Psi_{B}=r^{3 / 4} \Psi_{0}(\phi)+\Psi_{1}(\phi)+\cdots
$$

where the polar coordinates, $r$ and $\phi$, are defined as

$$
x=r \cos \phi, \quad y=r \sin \phi .
$$

If $F_{0}(\infty)$ is denoted by $A_{0}$ then, as shown by Yang \& Jerger [9], $\Psi_{0}$ satisfies

$$
\nabla^{2} \Psi_{0}=0
$$

subject to

$$
\Psi_{0}(\phi=0)=A_{0}, \quad \Psi_{0}(\phi=\alpha)=0,
$$

where the inhomogeneous boundary condition represents an asymptotic matching of $v$ with the boundary layer solution. The solution of Eq. (26) can be written as

$$
\Psi_{0}=-A_{0} \frac{\sin \frac{3}{4}(\phi-\alpha)}{\sin \frac{3}{4} \alpha} .
$$

We note, for later reference, that this solution is singular when $\alpha=4 \pi / 3$. On expanding Eq. (28) around $\phi=0$ and rewriting the result in terms of the similarity variable, $\eta$, it follows that the behaviour of $\Psi_{0}$ in the matching region is given by

$$
\Psi_{0} \sim A_{0}\left(1-\frac{3}{4} \eta x^{-3 / 4} \cot \frac{3}{4} \alpha+\cdots\right) .
$$

The second term in (29) provides the matching condition for the second streamfunction term in the boundary layer expansion, (18).

As shown by Yang $\&$ Jerger [9], the equations for $F_{1}$ and $H_{1}$, the first order boundary layer terms, are

$$
\begin{gathered}
F_{1}^{\prime \prime \prime}+\frac{3}{4} F_{0} F_{1}^{\prime \prime}-\frac{1}{4} F_{0}^{\prime} F_{1}^{\prime}+H_{1}=0 \\
H_{1}^{\prime \prime}+\frac{3}{4} \operatorname{Pr}\left(F_{0} H_{1}\right)^{\prime}=0
\end{gathered}
$$

subject to

$$
F_{1}(0)=F_{1}^{\prime}(0)=0, \quad H_{1}(0)=0, \quad F_{1}^{\prime}(\infty)=-\frac{3}{4} A_{0} \cot \frac{3}{4} \alpha, \quad H_{1}(\infty)=0
$$


where the inhomogeneous boundary condition represents a matching with the $O\left(x^{-3 / 4}\right)$ term in Eq. (29). Numerical integration of Eqs. (30)-(32) at $\operatorname{Pr}=6.7$ results in the values

$$
F_{1}^{\prime \prime}(0)=-0.13908, \quad H_{1}^{\prime}(0)=0, \quad A_{1}=-2.0622
$$

where

$$
F_{1}(\eta) \sim-\frac{3}{4} \cot \frac{3}{4} \alpha\left[-\eta A_{0}+A_{1}\right] \quad \text { as } \quad \eta \rightarrow \infty .
$$

The corresponding values for air $(\operatorname{Pr}=0.7)$ are

$$
F_{1}^{\prime \prime}(0)=-0.39967, \quad H_{1}^{\prime}(0)=0, \quad A_{1}=-3.37412
$$

We note that

$$
H_{1}=0
$$

may be derived analytically from equation (31), and therefore there is no correction to the temperature field at this order.

\section{Linear stability analysis.}

In this section we develop the linear stability equations for the basic flow given above by substituting

$$
\begin{aligned}
& \psi(x, y, t)=\psi_{B}(x, y)+\epsilon \psi_{D}(x, y, t) \\
& \theta(x, y, t)=\theta_{B}(x, y)+\epsilon \theta_{D}(x, y, t),
\end{aligned}
$$

into Eqs. (10) and (11). It is important to note that the asymptotic theory of the last section is being used to obtain what is hoped to be a closer approximation to the basic flow that is provided by the leading order boundary layer solution. On taking $|\epsilon| \ll 1$ and linearising we obtain the following system of linearised disturbance equations in streamfunction/vorticity form:

$$
\begin{gathered}
\nabla^{2} \psi_{D}=\omega_{D} \\
\nabla^{2} \omega_{D}=\frac{\partial \omega_{D}}{\partial t}+\frac{\partial \psi_{B}}{\partial y} \frac{\partial \omega_{D}}{\partial x}+\frac{\partial \psi_{D}}{\partial y} \frac{\partial \omega_{B}}{\partial x}-\frac{\partial \psi_{B}}{\partial x} \frac{\partial \omega_{D}}{\partial y}-\frac{\partial \psi_{D}}{\partial x} \frac{\partial \omega_{B}}{\partial y}-\frac{\partial \theta_{D}}{\partial y} \\
\frac{1}{P r} \nabla^{2} \theta_{D}=\frac{\partial \theta_{D}}{\partial t}+\frac{\partial \psi_{B}}{\partial y} \frac{\partial \theta_{D}}{\partial x}+\frac{\partial \psi_{D}}{\partial y} \frac{\partial \theta_{B}}{\partial x}-\frac{\partial \psi_{B}}{\partial x} \frac{\partial \theta_{D}}{\partial y}-\frac{\partial \psi_{D}}{\partial x} \frac{\partial \theta_{B}}{\partial y}
\end{gathered}
$$

where $\omega_{B}=\psi_{B y y}$ represents the vorticity corresponding to the basic flow. The appropriate boundary conditions to solve the disturbance equations are

$$
\begin{aligned}
& \psi_{D y}=\psi_{D}=0, \quad \theta_{D}=0 \quad \text { at } \quad y=0, x \geq 0 \\
& \psi_{D y} \rightarrow 0, \quad \omega_{D} \rightarrow 0 \quad \theta \rightarrow 0 \quad \text { as } \quad y \rightarrow \infty
\end{aligned}
$$


We now impose the parallel flow approximation where we assume that the disturbances have solutions of the form,

$$
\left(\begin{array}{c}
\psi_{D} \\
\omega_{D} \\
\theta_{D}
\end{array}\right)=\left(\begin{array}{c}
F(y) \\
G(y) \\
H(y)
\end{array}\right) e^{(i k x+\lambda t)}
$$

where the amplitudes $F(y), G(y)$, and $H(y)$ are the complex functions of $y$. Here $k$ is a real positive quantity and represents the wave number of the disturbance, and $\lambda=$ $\lambda_{R}+i \lambda_{I}$ is the complex temporal growth rate. Neutral stability corresponds to $\lambda_{R}=0$ and the computed value of $\lambda_{I}$ is related to the wavespeed of the disturbance which is $-\lambda_{I} / k$. The neutral stability condition $\lambda_{R}=0$ leads to a relation between $k$ and $x$ in the form of a curve in the $(k, x)$ plane and which is referred to as the neutral curve.

Eq. (43) may now be substituted into Eqs. (38)-(40), and on changing the variable from $y$ to $\eta$, the disturbance equations take the forms,

$$
\begin{gathered}
F^{\prime \prime}-k^{2} x^{1 / 2} F=x^{1 / 2} G \\
G^{\prime \prime}+\frac{\partial \psi_{B}}{\partial x} x^{1 / 4} G^{\prime}-\left[k^{2}+i \lambda_{I}+i k \frac{\partial \psi_{B}}{\partial y}\right] x^{1 / 2} G=\frac{\partial \omega_{B}}{\partial x} x^{1 / 4} F^{\prime}-i k \frac{\partial \omega_{B}}{\partial y} x^{1 / 2} F-x^{1 / 4} H^{\prime} \\
H^{\prime \prime}+\operatorname{Pr} \frac{\partial \psi_{B}}{\partial x} x^{1 / 4} H^{\prime}-\left[k^{2}+i \operatorname{Pr} \lambda_{I}+i k \operatorname{Pr} \frac{\partial \psi_{B}}{\partial y}\right] x^{1 / 2} H=\operatorname{Pr}\left[\frac{\partial \theta_{B}}{\partial x} x^{1 / 4} F^{\prime}-i k x^{1 / 2} \frac{\partial \theta_{B}}{\partial y} F\right]
\end{gathered}
$$

where primes represent derivatives with respect to $\eta$. The boundary conditions to be satisfied by the disturbances are that

$$
F^{\prime}=F=H=0 \quad \text { at } \quad \eta=0 \quad \text { and } \quad F, G, H \rightarrow 0 \quad \text { as } \quad \eta \rightarrow \infty .
$$

As this homogeneous system forms an eigenvalue problem for $x$ and $\lambda_{I}$ in terms of the wavenumber, $k$, an additional (complex) normalisation condition at $\eta=0$ is required to solve the disturbance equations and it is taken to be

$$
H^{\prime}=1
$$

This extra boundary condition means that we may solve Eqs. (44)-(48) and determine the eigenvalues, $x$ and $\lambda_{I}$. A suitably modified version of the Keller-box method is used to solve this ordinary differential eigensystem and it is discussed in the next section.

\section{Numerical method.}

In this section the numerical solutions of the basic flow Eqs. (21)-(23), and (30)-(32) and the disturbance Eqs. (44)-(48) are discussed. The equations for the basic flow were solved using a standard shooting method which employs Newton-Raphson iteration techniques. 
In this method the ordinary differential Eqs. (21), (22), (30) and (31) are written as a first order system of ordinary differential equations and a 4 th order Runge-Kutta method was employed to solve them. These solutions are accurate to at least six significant figures.

When solving the disturbance equations it was observed that the vorticity, $G$, and temperature function, $H$, both decay to zero very quickly as $\eta$ increases, but that the streamfunction, $F$, does not. Therefore we introduced the alternative boundary condition

$$
F^{\prime}+k x^{1 / 4} F \rightarrow 0 \quad \text { as } \quad \eta \rightarrow \infty
$$

which allows the streamfunction to exhibit the appropriate decay rate well before the streamfunction attains small values. Thus we were able to solve the problem on a smaller computational domain than would otherwise be necessary. All these disturbance equations are in the complex form, and after resolution into their real and imaginary parts we have six second order ordinary linear differential equations together with two eigenvalues.

Due to the stiffness of the eigensystem (especially for large values of $k x^{1 / 4}$ ) the RungeKutta method was abandoned in favour of a direct method. Thus a modified version of the Keller-box code was employed to solve the disturbance equations. For this type of method it is not necessary always to insist that the governing equations are reduced to first order form, and the present code solves the six second order differential equations using straightforward second order accurate central difference approximations. When the difference equations are suitably arranged the presence of two eigenvalues, $x$ and $\lambda_{I}$ causes the Jacobian matrix of Newton-Raphson iteration scheme, which is a central part of the Keller box methodology, to have two extra rows and columns over and above its usual block tridiagonal structure. Therefore the block-Thomas algorithm had to be modified to account for this structural change; very similar schemes were used by Lewis et al [10] and Shu and Wilkes [11].

\section{Numerical results.}

We present first the stability calculations for water for which we take $\operatorname{Pr}=6.7$. The detailed stability results in the form of $x-k$ neutral curves are shown in Figs. 2 and 3 where the different curves correspond to different values of the wedge angle. Here we see that there are very substantial variations in the shapes of the curves and that the minimum value of $x$ also varies considerably as $\alpha$ varies. In these figures we use a scaled value of $\alpha$ defined according to

$$
\gamma=\frac{12 \alpha}{\pi}
$$

Figure 2 shows curves corresponding to the range $0<\gamma<8$ (i.e. $0<\alpha<\frac{2}{3} \pi$ ), or, equivalently, $16<\gamma<24$ (i.e. $\frac{4}{3} \pi<\alpha<2 \pi$ ). Those corresponding to the intermediate range, $8<\gamma<16$ (i.e. $\frac{2}{3} \pi<\alpha<\frac{4}{3} \pi$ ) are displayed in Fig. 3. We note that the curve 
which corresponds to taking only the leading order boundary layer flow as the basic flow is obtained when $\cot 3 \alpha / 4=0$, i.e. when $\alpha=2 \pi / 3$ and $2 \pi$ (or $\gamma=8,24$ ). Conversely, the asymptotic analysis for the basic flow breaks down when cot $3 \alpha / 4$ is infinite, that is, for $\alpha=0, \frac{4}{3} \pi$ or $\gamma=0,16$.

When the wedge angle is $\alpha=\frac{2}{3} \pi(\gamma=8)$ the neutral stabiility curve is unimodal and displays the usual characteristic for boundary layer flows of having a maximum wavenumber for which instability may be expected. As the wedge angle decreases from this value towards zero the curve rises and the critical distance increases. The reason for this may be understood in terms of the effect of the second term in the basic vertical velocity which is negative, and increasingly so as $\alpha$ decreases. If, crudely speaking, we were to assume that instability takes place when the maximum streamwise velocity within the boundary layer attains a certain threshold value, then the decrease in this velocity due to the second term in the basic flow boundary layer analysis means that a greater distance from the leading edge is required before instability can take place.

On the other hand, when $\alpha$ increases from $\frac{2}{3} \pi$, the second term in the boundary layer flow increases the upwards velocity making the flow more susceptible to instability. This is seen quite clearly in Fig. 3, where the neutral curve is also found to attain a more complicated bimodal form. In this case, as $\alpha \rightarrow \frac{4}{3} \pi$, the singular case mentioned above for which the asymptotic expansion breaks down, the critical value of $x$ approaches zero.

The results from Figs. 2 and 3 are summarised in Fig. 4 in which is displayed the variation with $\gamma$ of both the local extrema of the neutral curves. We see that there is very little variation of the critical value of $x$ over most of this range, thereby lending confidence to analyses based only on the leading order boundary layer flow. However, anomalous results occur in the region fairly close to $\alpha=\frac{4}{3} \pi$ due to the incipient breakdown of the asymptotic analysis. It highly likely that a much more accurate representation of the basic flow will be required for such cases, and this may very well need to involve a full solution of the elliptic governing equations.

Equivalent results for the case of air, for which $\operatorname{Pr}=0.7$, are shown in Figs. 5 to 7 . Again the neutral curve corresponding to the leading order boundary layer flow is given by the case $\gamma=8$ in Fig. 5, and the curve is unimodal. As $\gamma$ decreases towards zero the neutral values of $x$ increase in general, but the detailed evolution is complicated by the fact that the neutral curve becomes bimodal. When $\gamma$ is close to 8 the left hand minimum corresponds to the smaller minimum, whereas the right hand one assumes this dominance as $\gamma$ decreases.

Fig. 6 shows that the neutral values of $x$ decrease towards zero as $\gamma$ increases towards 16 ; in this regard the behaviour is identical to that shown in Fig. 3. However, the curves assume increasingly bizarre shapes as $\gamma$ increases. 
The overall variation in the extrema of the neutral curves with $\alpha$ is given in Fig. 7. In the ranges $1<\gamma<5$ and $17<\gamma<21$ (i.e. $\frac{1}{12}<\alpha / \pi<\frac{5}{12}$ and $\frac{17}{12}<\alpha / \pi<\frac{21}{12}$ ) there is very little variation in the critical value of $x$. But outside of these ranges there is a very strong variation suggesting that the thermal boundary layer flow of air is particularly sensitive to the external geometry.

\section{Discussion and conclusions.}

Over much of the range of wedge angles the criterion for the onset of wave convection in water does not vary greatly from that found when using only the leading order basic flow. However, when the wedge angle is close to $4 \pi / 3$ (or to 0 ) the results become unreliable due to the fact that the second term in the asymptotic expansion becomes unbounded and the asymptotic expansion ceases to be uniformly valid. For wedge angles less than this critical value, the flow is increased in strength relative to the leading order flow and instability is enhanced, whereas the opposite effect is found at wedge angles slightly greater than $4 \pi / 3$. For air the neutral distance is much more sensitive to changes in $\alpha$ than for water.

The equivalent analysis for vortex instabilities in convective boundary layers in porous media was undertaken by Storesletten \& Rees [8] who also found a very substantial variation in the critical distance with wedge angle. In that context higher order terms in the basic flow become infinite only as $\alpha \rightarrow 0^{+}$. However, the conclusion there is that the variation in the value of $x_{c}$ with $\alpha$ is too great for the theory to be deemed reliable. This is due to the fact that the basic flow is not sufficiently well-represented by the three-term asymptotic expansion used in [8].

More generally it is very likely to be true that the position of onset of convection depends on the shape of the bounding region of the fluid. To support this contention we cite the experimental studies of Yan and Tao [12] and Yan and Zhang [13]. These authors consider an air-filled tall rectangular domain, and they obtain the respective critical Grashof numbers, 65 and 40, which are equivalent to $x_{c}=65$ and 34 here. These values are smaller than most of those depicted in Fig. 7. One possible explanation of this might lie with a comment made by Brooker et al [14] that self-sustaining oscillations have been observed in differentially heated cavities. In such situations a disturbance placed near the base of the hot vertical surface decays at first, but then begins to grow once it is past the neutral location. Thereafter it decays after turning the corner at the top of the cavity, continues to decay on beginning the descent of the cold vertical surface, but then grows as it did on ascending teh ht surface. Finally it decays once more as it passes along the lower surface and finally enters the hot boundary layer again in order to repeat the cycle. Therefore at critical conditions a neutrally stable feedback loop is established. This is, of course, an entirely different mechanism by which the shape of the 
domain affects the stability criterion, but it nevertheless shows that consideration must be given to conditions which are external to the boundary layer.

For the present flow, then, we would recommend (i) that the stability characteristics should be studied using a more accurate basic flow and (ii) that the role played by the overall domain chosen to represent the fluid dynamical situation be emphasized. Both of these recommendations involve the computation of a steady elliptic system of partial differential equations for the basic flow which is of equal difficulty to a detailed unsteady simulation of the full equations, and therefore a direct fully numerical approach should be adopted. Such work has been undertaken by the present authors for a thermal boundary layer in a completely unbounded fluid, and is in the process of being prepared for publication (Paul et al [15.16]).

\section{Acknowledgements}

The first author would like to acknowledge the contribution of an Overseas Research Studentship (ORS) and a University of Bath Studentship.

\section{References}

[1] B. Gebhart, Buoyancy induced fluid motions characteristic of applications in technology, Trans. A.S.M.E. J. Fluid Engineering 101 (1979) 5-28.

[2] P.R. Nachtsheim, Stability of free convection boundary layer flows, NASA TN D-2089 (1963).

[3] C.A. Heiber, B. Gebhart, Stability of vertical natural convection boundary laters, J. Fluid Mech. 48 (1971) 625-646.

[4] S.E. Haaland, E.M. Sparrow, Wave instability of natural convection on inclined surfaces according for nonparallelism of the basic flow, J. Heat Transfer 95 (1973) 405-407.

[5] F. Godaux, B. Gebhart, An experimental study of the transition of natural convection flow adjacent to a vertical surface, Int. J. Heat Mass Transfer 17 (1974) 93-107.

[6] A.A. Szewczyk, Stability and trasition of the free-convection layer along a vertical flat plat, Int. J. Heat Mass Transfer 5 (1962) 903-914.

[7] S.E. Haaland, E.M. Sparrow, Stability of buoyant boundary layers and plumes, taking account of nonparallelism of the basic flows, J. Heat Transfer 95 (1973) 293-301.

[8] L. Storesletten, D.A.S. Rees, The influence of higher-order effects on the linear instability of thermal boundary layer flow in porous media, Int. J. Heat Mass Transfer 41 (1998) 1833-1843.

[9] K.T. Yang, E.W. Jerger, First-order perturbations of laminar free-convection boundary layers on a vertical plate, J. Heat Transfer 86 (1964) 107-115. 
[10] S. Lewis, A.P. Bassom, D.A.S. Rees, The stability of vertical thermal boundary layer flow in a porous medium, Eur. Journal Mech. B: Fluids 14 (1995) 395-408.

[11] J.J. Shu, G. Wilkes, Mixed convection kaminar film condensation on a semi-infinite vertical plate, J. Fluid Mech. 300 (1995) 207-229.

[12] D. Yan, J.J. Tao, An experimental research on the instability of natural convection boundary layer around a vertical heated plate, Acta Mechanica Sinica (English Series) 15 (1999) 1-7.

[13] D. Yan, H.X. Zhang, Buoyancy instability in the natural convection boundary layer around a vertical heated flat plate, Acta Mechanica Sinica (English Series) 18 (2002) 126-132.

[14] A.M.H. Brooker, J.C. Patterson, S.W. Armfield, Non-parallel linear stability analysis of the vertical boundary layer in a differentially heated cavity, J. Fluid Mech. 352 (1997) $265-281$.

[15] M.C. Paul, D.A.S. Rees, M. Wilson, Receptivity of free convective flow from a heated vertical surface. I. Linear waves, submitted for publication.

[16] M.C. Paul, D.A.S. Rees, M. Wilson, Receptivity of free convective flow from a heated vertical surface. II. Nonlinear waves, submitted for publication. 

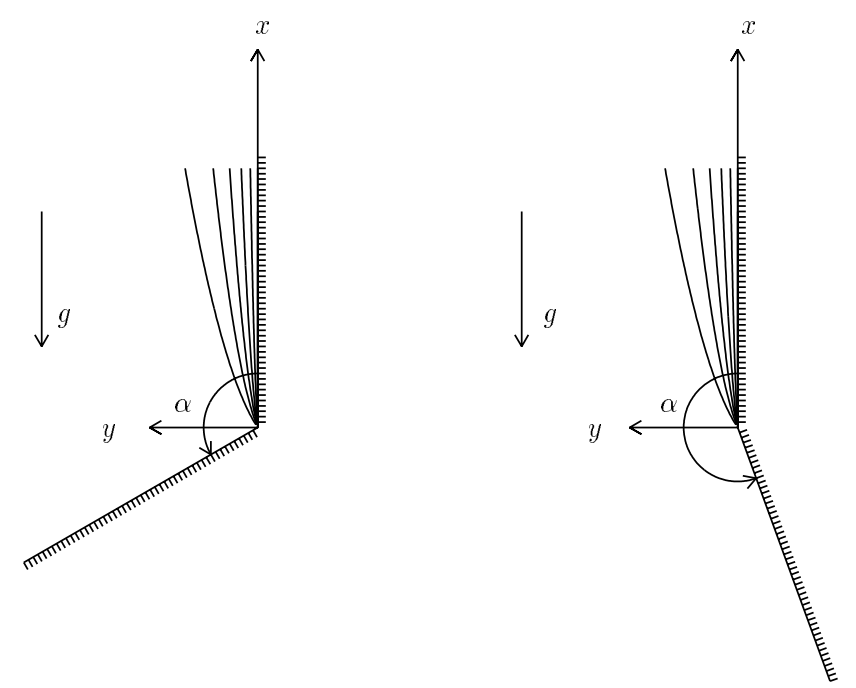

Fig. 1.

Schematic diagram of the flow configuration showing the coordinate directions, the orientation of surfaces, and displaying two different wedge-angles, $\alpha$. 


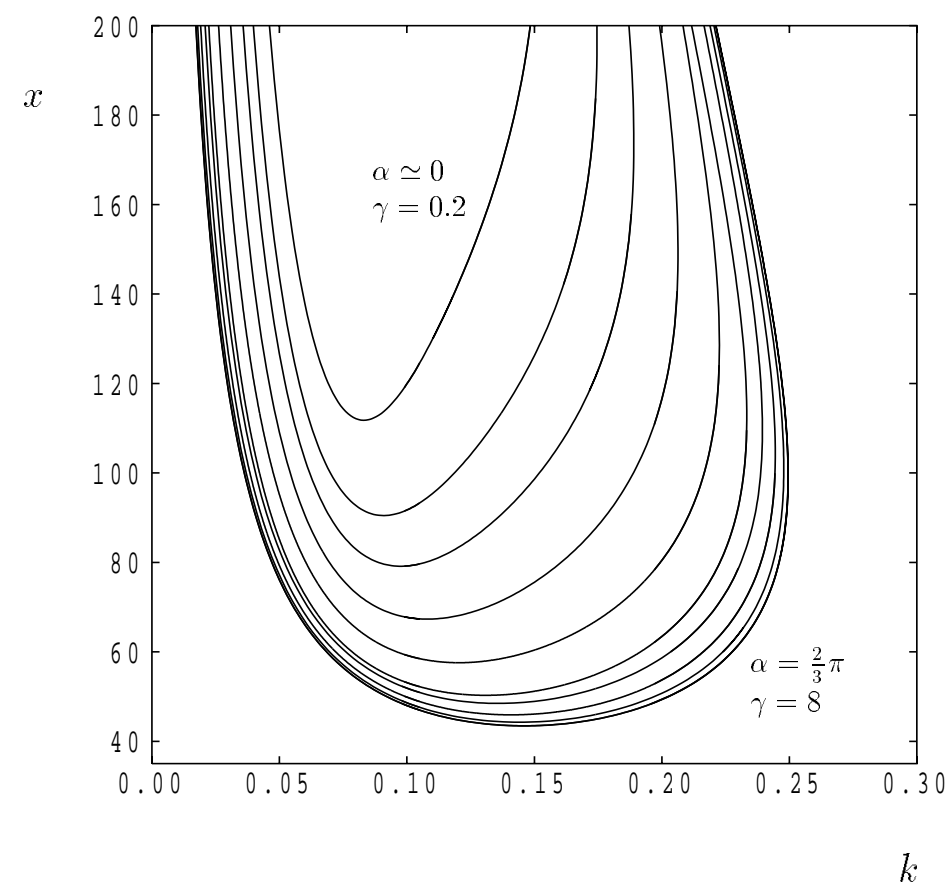

Fig. 2.

Neutral curves for water $(\operatorname{Pr}=6.7)$, corresponding to $\gamma=0.2,0.3,0.4,0.6,0.8,1,2,4,6,8$ where $\gamma=12 \alpha / \pi$. This corresponds to $\alpha$ varying between just above 0 and $\frac{2}{3} \pi$. 


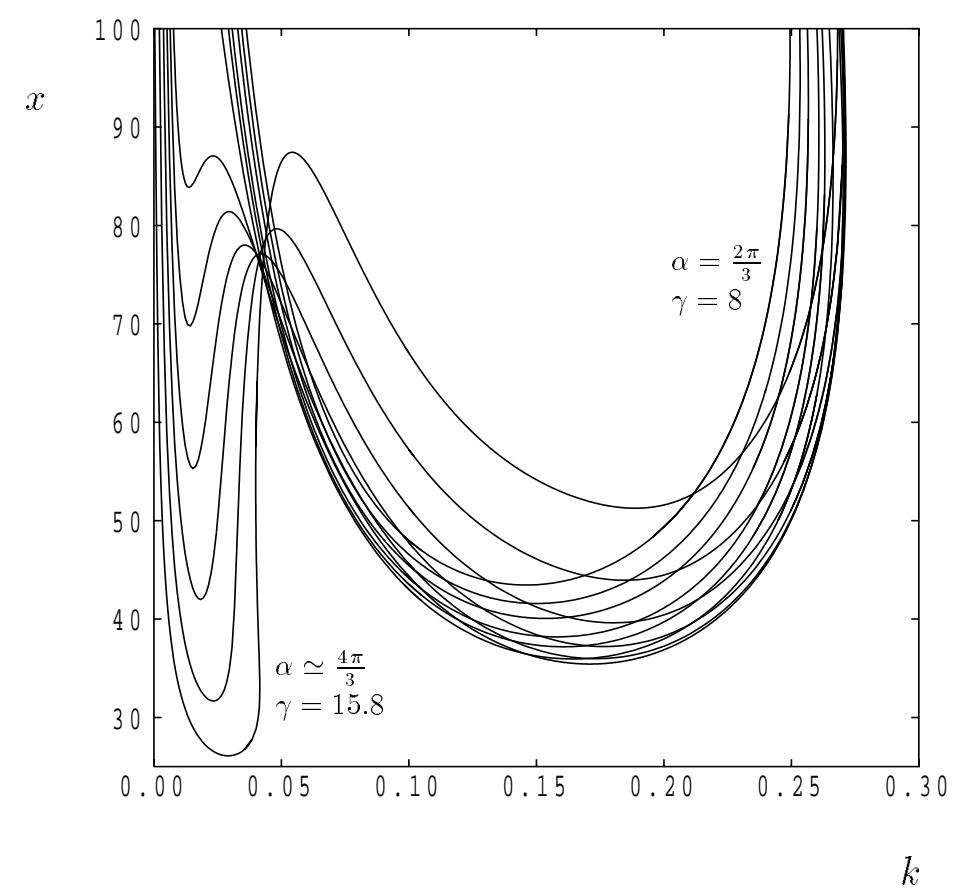

Fig. 3.

Neutral curves for water $(\operatorname{Pr}=6.7)$, corresponding to $\gamma=8,9,12,14,14.4,14.6,14.8,15,15.2,15.4,15.6$, 15.8 , where $\gamma=12 \alpha / \pi$. This corresponds to $\alpha$ varying between $2 \pi / 3$ and almost $4 \pi / 3$. 


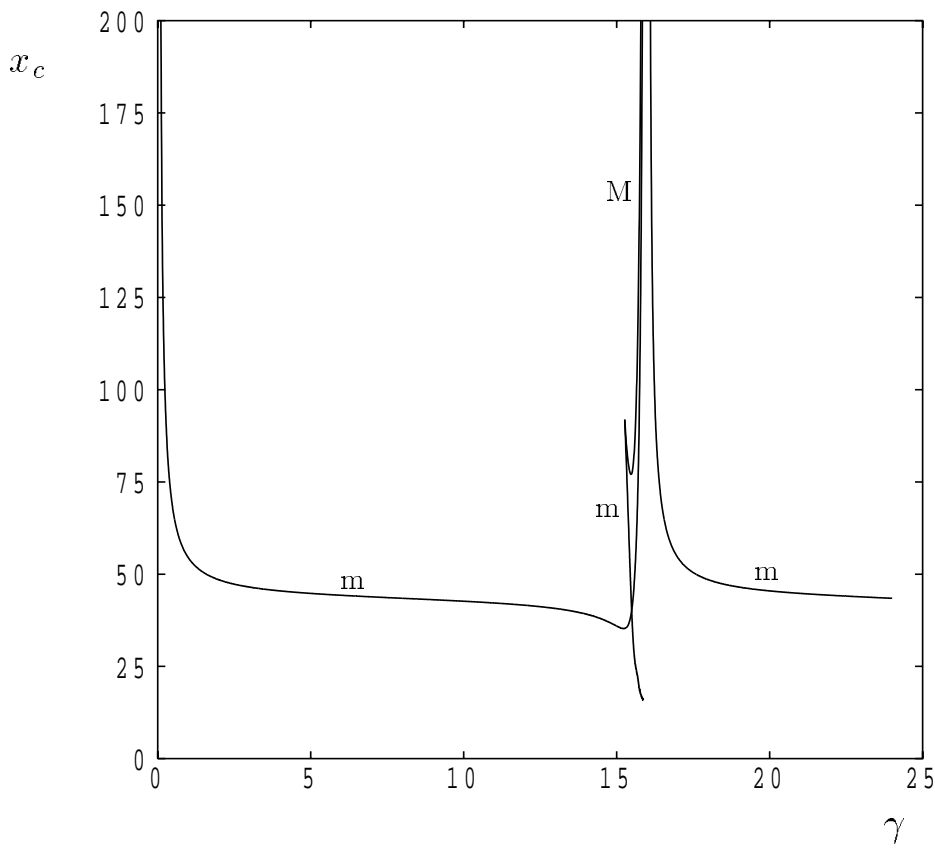

Fig. 4.

Evolution of the critical points on the neutral curves against $\gamma=12 \alpha / \pi$ for water $(\operatorname{Pr}=6.7)$. $M$ denotes a local maximum while $m$ denotes a local minimum. 


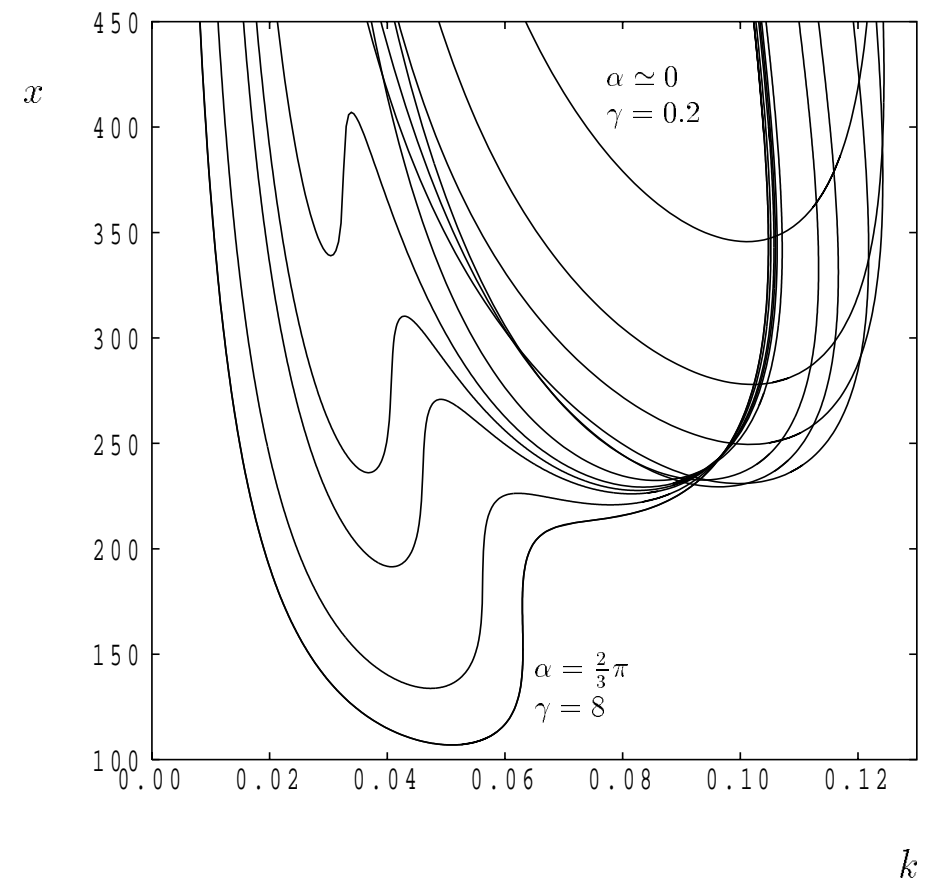

Fig. 5.

Neutral curves for air $(\operatorname{Pr}=0.7)$, corresponding to $\gamma=$ $0.2,0.3,0.4,0.6,0.8,1,2,4,6$ and 8 where $\gamma=12 \alpha / \pi$. This corresponds to $\alpha$ varying between just above 0 and $\frac{2}{3} \pi$. 


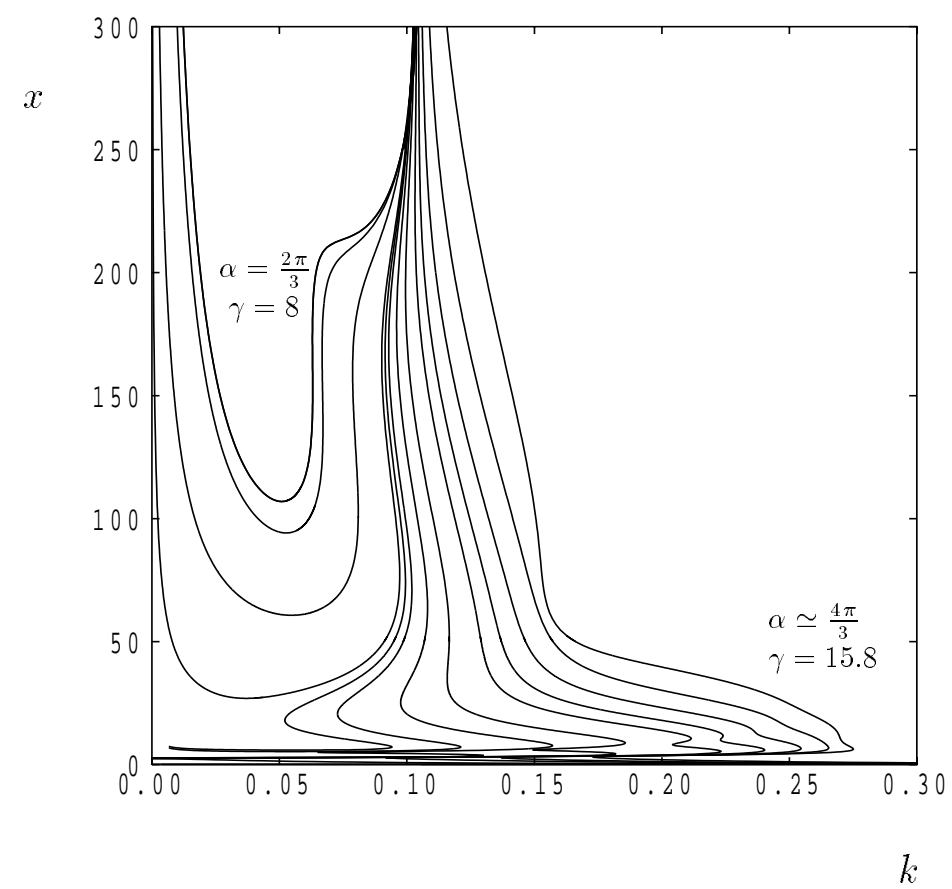

Fig. 6.

Neutral curves for air $(\operatorname{Pr}=0.7)$, corresponding to $\gamma=$ $8,9,12,14,14.2,14.4,14.6,14.8,15,15.2,15.4,15.6$, 15.8 , where $\gamma=12 \alpha / \pi$. This corresponds to $\alpha$ varying between $2 \pi / 3$ and almost $4 \pi / 3$. 


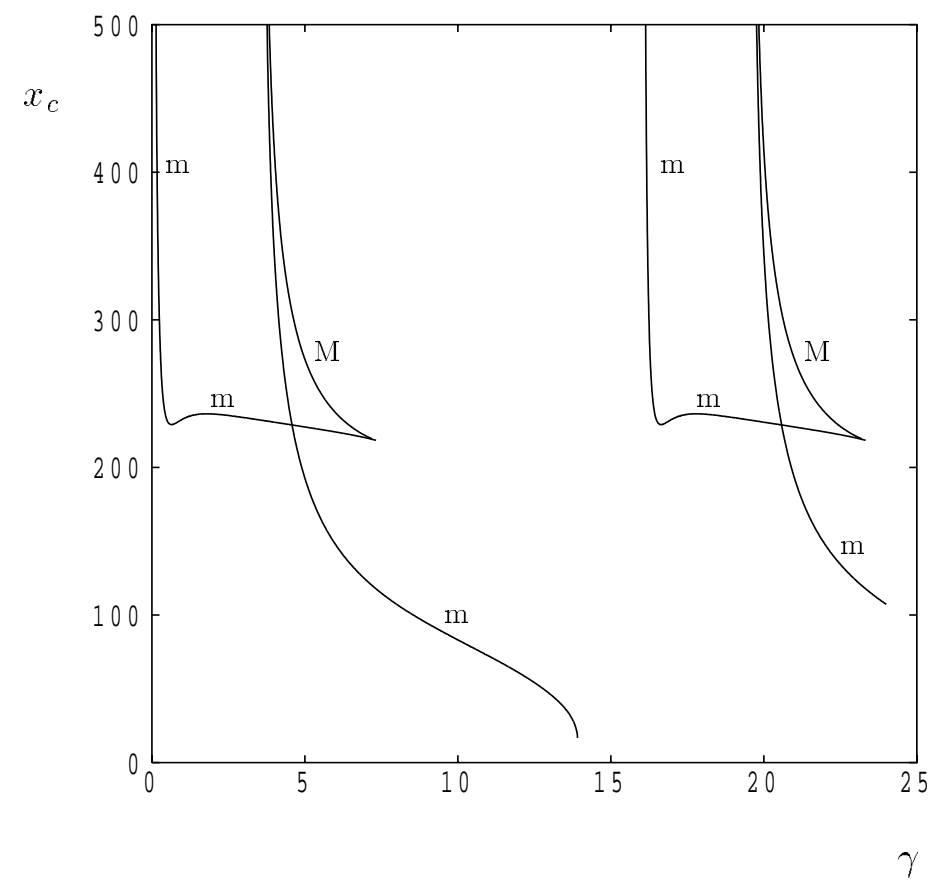

Fig. 7.

Evolution of the critical points on the neutral curves against $\gamma=12 \alpha / \pi$ for air $(P r=0.7)$. $M$ denotes a local maximum while $m$ denotes a local minimum. 